\title{
Promover la inclusión de las familias a través del desarrollo de Proyectos de Trabajo. Un estudio de caso
}

\section{Promoting the inclusion of families through the development of Work Projects. A case study}

\author{
María del Carmen RUIZ TORRES y Rosario MÉRIDA SERRANO \\ Universidad de Córdoba
}

Recibido: Noviembre 2014

Evaluado: Abril 2015

Aceptado: Mayo 2015

\section{Resumen}

Se presenta una investigación cualitativa centrada en un estudio de caso realizado en un aula de niños y niñas de 5 años. Se analiza la potencialidad de la metodología de Proyectos de Trabajo (en adelante, PT) para facilitar la inclusión de las familias en el proceso de enseñanzaaprendizaje de sus hijos e hijas. A través de tres entrevistas en profundidad a las familias y de la documentación pedagógica obtenemos información que, tras ser analizada, revela los siguientes hallazgos: (1) La metodología de PT utiliza como parte esencial del proceso de enseñanzaaprendizaje los conocimientos y la experiencia profesional de las familias; (2) Las familias asumen un papel de tutorización y acompañamiento en los procesos educativos de sus hijos e hijas que, de forma simultánea, se producen en la escuela y en sus casas. Ajustan sus intervenciones a las demandas y realizan una labor de mediación entre las capacidades infantiles y el nuevo material de aprendizaje; (3) La colaboración de las familias, presente desde el inicio hasta que concluye el PT, requiere un compromiso muy elevado, resultando ser un elemento imprescindible para el desarrollo de esta metodología; y (4) Las familias identifican como fortaleza de los PT la escucha al alumnado. Como limitaciones, el tiempo desmesurado que requiere, las diferencias que puede generar en el alumnado los diferentes niveles de implicación de sus respectivas familias y la discontinuidad de este método con otras formas más tradicionales de afrontar la enseñanza en etapas posteriores.

Palabras clave: proyectos de trabajo, educación inclusiva, diversidad, educación infantil, familia, estudio de caso.

\footnotetext{
Abstract

This is a qualitative research focused on a case study in a classroom of 5-years-old children. The potential of the Work Projects (hereinafter PT) methodology is analysed to facilitate the inclusion of families in the teaching-learning process of their children. Through three detailed interviews to the families and pedagogical documentation, we obtain information which -after the analysis- reveals the following findings: (1) PT methodology uses as an essential part of the teaching-learning process the knowledge and professional experience of families; (2) Families assume a role of tutoring and support in the educational process of their children, which occur 
simultaneously at school and at home. They adjust their actions to the demands and carry out the task of mediating between children's skills and learning new material; (3) The collaboration of families, present from the beginning to the end of the PT, requires a very high commitment, proving to be an indispensable element for the development of this methodology; and (4) Families identify listening to students as a strength of the PT. As limitations, the excessive time required, the differences which can generate in students the different levels of involvement of their families and the discontinuity of this method with more traditional ways of dealing with teaching at later stages.

Keywords: work projects, inclusive education, diversity, childhood education, family, case study.

Esta investigación se enmarca en la red RIECU ${ }^{1}$ (Red de Infantil, Escuela-Centro de Formación del Profesorado-Universidad). Se trata de una red de profesionales construida para fortalecer la aplicación de la metodología de PT y propiciar la continuidad entre la formación docente inicial y permanente. En este trabajo focalizamos nuestra atención en la capacidad de los PT para favorecer la inclusión de la diversidad de las familias en el proceso de enseñanza-aprendizaje que se desarrolla al poner en marcha un PT en un aula de alumnado de cinco años. La investigadora permanece dos meses en la clase, asumiendo el rol de observadora participante mientras recoge información. Los instrumentos utilizados (entrevistas, observación sistemática y documentación pedagógica) permiten obtener datos de naturaleza textual y visual, al tiempo que actúan como fuentes de triangulación para incrementar la consistencia de los hallazgos alcanzados. El análisis del discurso y de contenido utilizados para analizar ambos tipos de datos nos permiten concluir que las familias son incluidas al aplicar la metodología de PT en el aula. Su implicación está presente desde la elección del tema, así como en las diferentes fases del PT -documentación, producción y evaluación-. Finalmente, se analizan algunas debilidades y sugerencias que pudieran contribuir a mejorar la implementación de esta metodología en las aulas infantiles.

\section{La educación inclusiva como respuesta a la diversidad familiar en educación infantil}

La diversidad es una característica que nos hace a las personas únicas (Aguado Odina, 2010). Las diferencias individuales han de ser respetadas desde el entorno educativo, puesto que la educación ha de entenderse como el derecho que tiene toda persona a poseer las mismas oportunidades de desarrollo Sin embargo, no podemos confundir el término igualdad educativa con la homogeneidad escolar frecuente en muchas aulas ni el de desigualdad con el de diferencia (Muntaner, 2010).

\footnotetext{
${ }^{1}$ Este trabajo se desarrolla en el aula de la maestra Ana López, al amparo de un proyecto interinstitucional aprobado por la Delegación Provincial de Educación de Córdoba y el Vicerrectorado de Estudios de Postgrado y Formación Continua de la Universidad de Córdoba, 2012/13.
} 
En el contexto educativo, la diversidad familiar debe abordarse desde una perspectiva amplia, concediéndole un alto valor educativo (Benito y Ballesteros, 2012). Dar respuesta a la diversidad familiar supone incorporar las diferencias y aprovecharlas para enriquecer el proceso educativo que tiene lugar en las aulas infantiles (Arnáiz y De Haro, 2004). Una forma de respetar la diversidad familiar es poner en marcha procesos de inclusión.

La educación inclusiva aboga por una escuela que reduzca las barreras presentadas al alumnado y ofrece los recursos necesarios que este requiere. Es conceptuada como un valor del que se beneficia toda la comunidad educativa (Parrilla, 2002). Como señalan Echeíta y Sandoval (2002) la educación inclusiva se puede definir como un 'movimiento' que aglutina a muchas corrientes teóricas y prácticas educativas que comparten un denominador común: (1) respetar la idiosincrasia y diferencias de todo el alumnado, ampliando la visión reduccionista y sesgada de identificar las diversidad con la atención a las necesidades específicas de apoyo; (2) optar por una educación basada en un currículum flexible, que se apoya en metodologías activas, cooperativas, plurales y personalizadas; y (3) abrir los procesos educativos a las aportaciones y participación de la comunidad.

Esta necesidad de inclusión, necesaria en cualquier etapa del sistema educativo, resulta imprescindible en la etapa infantil al ser el primer contexto de socialización en el que el niño o la niña participa, más allá del escenario familiar. Su perfil personal único ha sido atendido de forma prioritaria en el seno de su familia. Dicha atención familiar, empapada por un vínculo de apego consistente, constituye su universo personal antes de llegar a la escuela. Al encontrarnos en una etapa donde se configura la identidad personal, debemos evitar rupturas y discontinuidades en el tránsito a la experiencia educativa institucionalizada. Por tanto, la diversificación de estrategias metodológicas, el juego, la creación de un clima de afecto y seguridad, el mantenimiento de un estrecho vínculo entre quien educa y los niños y niñas, la atención personalizada basada en el conocimiento, la aceptación y el respeto a las diferencias personales, la participación de la comunidad, y en especial de las familias, son elementos necesarios para responder al derecho de una educación de calidad en la infancia (Booth, Ainscow y Kingston, 2006).

Como se deriva del párrafo anterior, una escuela inclusiva para la infancia no surge de un día para otro, sino que es una inversión a largo plazo que necesita ir consolidándose paulatinamente mediante un cambio de la cultura organizativa $\mathrm{y}$ profesional docente, a través de la introducción de prácticas inclusivas y poniendo en marcha acciones de formación continua que favorezcan procesos de reflexión colaborativa para repensar el sentido de la escuela, la igualdad de oportunidades, el rol docente y la finalidad de los procesos educativos, entre otros. Supone un cambio profundo del sistema educativo en el que es necesario un gran esfuerzo de participación de todos los sectores de la comunidad educativa, así como una nueva concepción del currículum y de los valores que se transmiten (Ainscow, Booth y Dyson, 2004 y Guijo, 2008). 


\section{La pluralidad familiar en la sociedad actual}

Tradicionalmente, en el ámbito educativo, Ha existido una escasa preocupación para desarrollar contenidos referidos a la diversidad familiar existente en Educación Infantil. En su lugar, el currículo ha ofrecido contenidos referidos a un tipo de familia estándar, muy diferente a los modelos de familias reales de los aprendices. (monoparentales, reconstituidas, ensambladas, homoparentales...) propias de una sociedad plural (López, Díez, Morgado y González, 2008).

Hace solo unas décadas el entorno familiar de los niños y niñas de Educación Infantil era muy diferente al actual, existiendo unos roles intrafamiliares bien definidos que practicaban estilos de vida semejantes. Por un lado, las madres se ocupaban de las tareas del hogar, mientras que el padre era el sustentador de la economía familiar. Ahora existen multitud de estilos familiares con distintas características. Se pueden apreciar diferentes estilos de crianza y modelos educativos familiares (democrático, autoritario y permisivo) que intensifican la diversidad de los niños y niñas en sus comportamientos, valores, actitudes, experiencias y modos de situarse en la escuela. Todo esto es reflejo de la diversidad social que existe (Aguado Iribarren, 2010 ).

Además de la diversidad de los perfiles familiares actuales, las familias más vulnerables son las que tienen mayor dificultad para propiciar el éxito escolar de sus hijos e hijas, reduciéndose de este modo el derecho a la igualdad de oportunidades que preconiza la Convención sobre los Derechos del Niño. (Guijo, 2008). Así, la escuela inclusiva debe estar construida sobre unos pilares institucionales consistentes a través de la cooperación y el sentimiento de pertenencia. De igual modo que la sociedad y la escuela, la familia es un agente socializador muy importante. Proporciona el cuidado y educación de los niños y niñas mediante una acción integrada adecuada que permite desarrollar las curiosidades, hábitos y disposiciones que harán que el aprendiz configure una personalidad y una forma de ser determinada (Ruiz de Miguel,1999 y Luengo y Luzón, 2001). El aprendizaje del alumnado depende, en gran medida, de la relación entre la escuela y la familia, así como de la consistencia con la que ambas agencias ejercen su acción formativa (Ainscow, Booth, y Dyson, 2004). De esta manera, escuela y familia están llamadas a trabajar conjuntamente y a hacer un esfuerzo compartido por incluir la diversidad familiar en el proceso educativo desarrollado en la etapa de Educación Infantil (Sánchez y García, 2009).

\section{Los proyectos de trabajo: una estrategia educativa de trabajo conjunto entre docente-alumnado-familia.}

Los PT es un modelo de aprendizaje basado en el enfoque de investigación que surge en la década de los años 80 ante la necesidad de dar al currículum un enfoque globalizado que despertara el interés del alumnado y que fuera válido para transferirlo fuera del contexto del aula, facilitando el abordaje de problemas de la vida real (Hernández, 2000).

Los PT tienen sus antecedentes en Kilpatrick y están fundamentados a partir del enfoque globalizador y la teoría socio-constructivista del aprendizaje, principalmente. 
Los PT demandan que la organización del currículum sea flexible, transdisciplinar y globalizada. La flexibilidad curricular se refiere a la capacidad del currículum de adaptarse a las diferentes situaciones y contextos, donde el tiempo y el espacio no es algo prefijado de antemano (Gallardo, 2013).Además, con un currículum transdisciplinar, se pretende el desarrollo de distintas temáticas elegidas por el alumnado, resultado de un diálogo de la realidad acerca de los cambios que se suceden en ella. En el modelo curricular que defienden los PT, no se distinguen asignaturas sino que, todos los conocimientos están globalizados (Sales, 2004). Mediante los PT, se pueden abordar los contenidos de las áreas del currículum de Educación Infantil y tener en cuenta los intereses y motivaciones del alumnado, favoreciendo así, el aprendizaje (Díez, 2002; Domínguez, 2003; Hernández, 2002 y Mérida et al., 2011).

También, los PT pretenden aportar funcionalidad al aprendizaje, haciendo que los contenidos sean de utilidad en su vida cotidiana. Es una forma de incluir la diversidad en la Educación Infantil, pues la escuela no es una realidad aparte, sino que debe existir permeabilidad y conexión entre la escuela y el mundo que rodea al alumnado (Hernández, 2002 y Zabala, 2012).

Las fases más significativas del método de PT, siguiendo las aportaciones de Mérida, González y Olivares (2015) son: (1) Situación desencadenante, donde se produce, bien de forma espontánea por parte de los niños y niñas, o bien inducido por la maestra, la elección del tema del PT: (2) Petición de colaboración a las familias para suministro de información, preparación de materiales, acompañamiento del alumnado; (3) Análisis de ideas previas a través de la asamblea y el dibujo sobre el tema del PT; (4) Planificación, junto con el alumnado de lo que queremos aprender, elaborando un guión de interrogantes a los que hay que dar respuesta en el proceso de investigación que se pone en marcha; (5) Consenso de lo que queremos hacer (actividades) para aprenderlo. En esta fase el alumnado y la docente proponen situaciones de aprendizaje para la documentación, la producción y la evaluación; y (6) Valoración final del PT.

Como se puede comprobar, los PT pretenden la participación del alumnado y del docente, y además, consideran de vital importancia la implicación de todos los miembros de la comunidad educativa, especialmente la colaboración de las familias (Domínguez, 2003 y Hernández, 2000). Sin ellas, difícilmente se podrían desarrollar los PT de forma eficaz, debido a la gran ayuda que prestan para la obtención de material y la búsqueda de información, ofreciendo sus experiencias vitales y profesionales, etc. También, las familias colaboran en la resolución de dudas, proponiendo descubrimientos y reflexionando durante el proceso de investigación que se desarrolla (Domínguez, 2003 y Mérida et al., 2011).

\section{Diseño del estudio}

\section{Contexto}

Esta investigación se desarrolla en un centro público de Educación Infantil y Primaria situado en una localidad cordobesa localizado a 50 kilómetros de la capital. Es un colegio que acoge a un voluminoso número de alumnado que poseen una gran 
diversidad sociocultural, ya que es el único centro público de la localidad. El aula en que desarrollamos la investigación es de tercer curso del segundo ciclo de la etapa de Educación Infantil.

\section{Participantes}

Los sujetos que participan son: 19 alumnos y alumnas (11 niños y 8 niñas) de 5-6 años, escolarizados desde los tres años en el mismo centro y que han seguido la metodología de PT, en su mayoría. También participa la docente que cuenta con una formación específica y gran experiencia en el desarrollo del método de PT. Finalmente, participan 19 familias con un nivel socioeconómico medio-bajo, siendo tres madres las entrevistadas para la recogida de información de la investigación.

\section{Objetivos}

El problema que aborda esta investigación se centra en analizar si la metodología de PT promueve la inclusión de la diversidad familiar en los procesos de enseñanzaaprendizaje.

Se concreta en los siguientes objetivos:

- Analizar los conocimientos y experiencias profesionales que las familias aportan al desarrollo del método de PT.

- Identificar si las familias asumen una tutorización o acompañamiento del aprendizaje de sus hijos e hijas al desarrollar el PT.

- Indagar la participación de las familias en el desarrollo de las actividades que forman parte del PT.

- Conocer las percepciones familiares sobre el método de PT, identificando fortalezas y debilidades.

\section{Método}

Nos encontramos ante una investigación cualitativa que se concreta en un estudio de caso. La investigadora permanece en un aula de alumnado de 5 años durante dos meses, asumiendo el rol de investigadora y observadora participante, y mantiene interacciones permanentes con los niños y niñas, la docente y las familias, como sujetos de investigación.

\section{Instrumentos}

La recogida de información en la investigación completa se realiza a través de diferentes instrumentos, prevaleciendo las entrevistas, la observación sistemática, y la 
documentación pedagógica. Para este trabajo se han seleccionado las entrevistas en profundidad realizadas a tres familias y la documentación pedagógica.

\section{Técnicas de análisis}

Para analizar los datos textuales derivados de las entrevistas en profundidad a las familias se utiliza la técnica de análisis del discurso. Por los datos visuales derivados de la documentación pedagógica se emplea el análisis de contenido (Banks, 2010).

En un primer análisis de los datos se construye una matriz hermenéutica (T1.) que incluye las dimensiones y categorías en las que se agrupan los datos. Su elaboración responde a un proceso mixto (deductivo e inductivo). Partimos de unas dimensiones macro derivadas del marco teórico, que se han enriquecido durante el trabajo de campo y mediante el análisis de los datos, al incorporar las categorías emergentes que van surgiendo. La complementariedad de los datos textuales y visuales, mediante un proceso de triangulación metodológica, permite incrementar la consistencia de los hallazgos obtenidos (Flick, 2004).

Para realizar el análisis del discurso de los datos textuales se utiliza el software informático Atlas.ti 6., a través de la matriz hermenéutica propuesta. Primero se realiza una clasificación de los datos, y posteriormente se lleva a cabo un proceso de codificación. Los datos visuales se asignan a la dimensión y categoría que corresponde según el contenido de los mismos, siguiendo las directrices que plantea Gibbs (2012).

\begin{tabular}{|c|c|}
\hline DIMENSIONES & CATEGORIAS \\
\hline 1. Concepto de diversidad & $\begin{array}{l}\text { 1.1. Connatural a la condición humana ("Todos } \\
\text { somos diversos"). }\end{array}$ \\
\hline 2. Tipos de diversidad & $\begin{array}{l}\text { 2.1. Diversidad de desarrollo y madurez } \\
\text { 2.2. Diversidad de ritmos de aprendizaje } \\
\text { 2.3. Diversidad de intereses y } \\
\text { motivaciones. } \\
\text { 2.4. Diversidad de origen sociocultural } \\
\text { 2.4.1. Diversidad de origen nacional } \\
\text { 2.4.2. Diversidad de origen } \\
\text { socioeconómico } \\
\end{array}$ \\
\hline 3. Estimulación del entorno familiar & $\begin{array}{l}\text { 3.1. Los estímulos familiares y la elección del } \\
\text { PT. } \\
\text { 3.2. Las ideas previas y experiencias familiares. }\end{array}$ \\
\hline 4. Inclusividad de las familias en el PT & $\begin{array}{l}\text { 4.1. Información del tema del PT } \\
\text { 4.2. Clarificación del papel de las familias } \\
\text { 4.3. Valoración de la colaboración familiar } \\
\text { 4.4. Implicación afectiva de las familias } \\
\text { 4.5. Aula abierta }\end{array}$ \\
\hline $\begin{array}{l}\text { 5. Co-responsabilidad familiar en el apren- } \\
\text { dizaje }\end{array}$ & $\begin{array}{l}\text { 5.1. Aportaciones familiares en: } \\
\text { 5.1.1. La fase de documentación } \\
\text { 5.1.2. La fase de producción } \\
\text { 5.1.3. La fase de evaluación }\end{array}$ \\
\hline 6. Valoración familiar de los PT & $\begin{array}{l}6.1 . \text { Beneficios } \\
\text { 6.2. Limitaciones } \\
\text { 6.3. Sugerencias }\end{array}$ \\
\hline
\end{tabular}

Tabla 1. Matriz Hermenéutica (T.1.) 


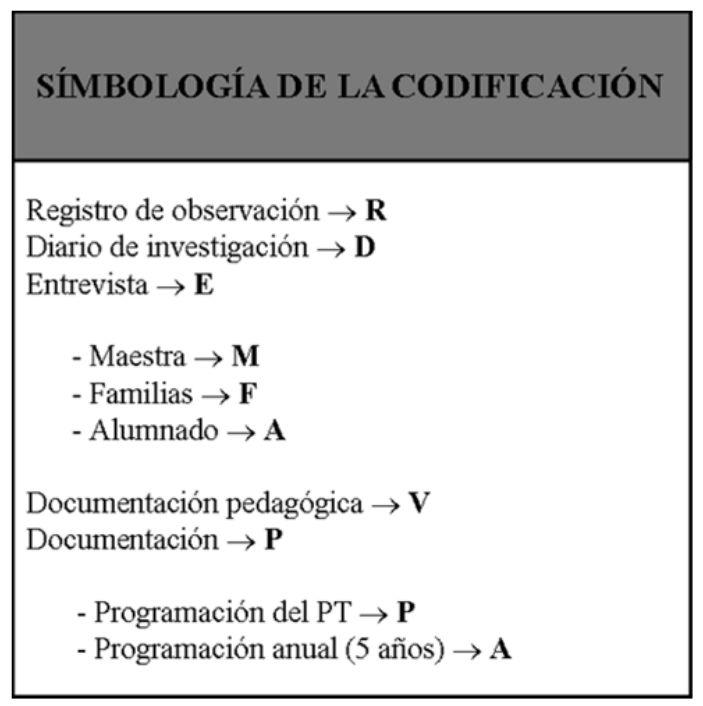

Tabla 2. Simbología de la codificación.

\section{Resultados}

En la primera dimensión, los resultados muestran que la docente entiende que la diversidad es un rasgo connatural de los seres humanos. Ella entiende la diversidad desde una concepción amplia, y la identifica con la diferencia y singularidad de cada persona. Además, considera la diversidad como una forma de enriquecimiento para el aula:

"Aunque hay diferencias, estas las aprovechamos para aprender más; no para marcar esa dificultad, sino para aprovecharla". (E.M.1.1.)

"La heterogeneidad es riqueza". (E.M.1.1.)

Respecto a la segunda dimensión, la docente es consciente de que existen distintos tipos de diversidad que caracterizan a las personas, referidos al desarrollo y madurez, a los ritmos de aprendizaje, a las motivaciones que tienen los niños y niñas y a la diversidad sociocultural de origen nacional y de origen socioeconómico. La maestra lo relata del siguiente modo:

"Nosotros tenemos niños que tienen inteligencias múltiples". (E.M.2.1.)

"No todos son muy expertos, digamos, en lectura o no todos, en creatividad, pero si hay unos que se les dan muy bien las matemáticas, otros que se les dan muy bien las manualidades....". (E.M.2.1.)

"Cuando hacemos un trabajo hay niños o niñas que pueden llegar más, otros que llegan hasta un cierto límite". (E.M.2.2.) 
"Hay niños que están mucho más entusiasmados que otros dependiendo de los temas, pero bueno eso nos pasa a los adultos también, ¿No?" (E.M.2.3.)

"Cuando vemos lo niños que tenemos que no son españoles, que son de otros paises, ellos te dicen, "pues bueno, pues yo en China he visto tal" y a lo mejor estamos investigando de Egipto, pero resulta que sale China y también se aportan cosas". (E.M.2.4.1.)

"Hay familias que pueden acceder a unos recursos y otras que no". (E.M.2.4.2.)

La tercera dimensión abarca la relación entre el entorno familiar y los PT. Los resultados muestran la influencia de los estímulos familiares en la elección del PT y de las ideas previas y experiencias familiares en el alumnado. El tema del proyecto desarrollado durante el trabajo de campo es Egipto. Se aprecia que la elección de dicha temática surge de una situación natural de clase en la que una alumna comienza a hablar de Egipto, y a sus compañeros y compañeras les despierta la curiosidad por investigar sobre este tema.

Como es habitual, se realiza la votación y la voluntad de la mayoría de los niños y niñas se inclina por elegir Egipto como tema a investigar. La maestra actúa en todo momento como mera mediadora de la situación:

"El Proyecto surge por la propuesta de una de las alumnas antes de terminar el anterior proyecto. Esta propuesta es secundada por el grupo" (P.P.3.1.)

"A los niños hay que marcarles unos límites, pero ellos saben que cuando hay situaciones en las que pueden opinar, se les va a preguntar. Y se enfadan cuando ya no les preguntas". (E.F.3.1.A.)

"Todo en la tele y en los libros resulta que es de lo que están dando. Hasta ella (hija), por ejemplo en los libros de su hermano, empieza a coger sus libros o va pasando. El otro día dio la casualidad que en el libro del hermano "mira mami el ojo de Horus" (...) Una cosa que los vemos motivados, además, que empieza a buscar en sus libros, o cuentos o lo que sea, y la ves a ver qué encuentra en los libros que tenga que ver con lo que están dando". (E.F.3.1.C.)

En los siguientes resultados se observa la influencia del entorno familiar sobre el alumnado durante el análisis de ideas previas y experiencias sobre el tema a tratar. Se puede comprobar cómo se incluyen las distintas ideas previas que el alumnado manifiesta. Además, tanto la maestra como los alumnos y alumnas conocen esta fase inicial del proyecto y se muestran muy dialogantes en ella:

"Después de acordar investigar sobre Egipto, pasamos a hacer una asamblea inicial sobre el tema y hablan sobre ¿Qué sabemos sobre Egipto?". (R.3.2.)

"Cada uno aporta sus experiencias y conocimientos respecto al tema". (P.A.3.2.) 
"Ellos conocen, por su entorno social, muchisimos datos sobre el día a día". (E.M.3.2.)

"Lo bueno es que, como el aula es un lugar donde ellos van contando su vida, sus experiencias y están acostumbrados a expresarse, pues es un lugar de puesta en común general y todo se acepta. La verdad que a nosotros nos beneficia y aprovechamos". (E.M.3.2.)

Haciendo referencia a la cuarta dimensión, los resultados muestran que la familia está incluida durante las diferentes fases de los PT. En primer lugar, se aprecia que las familias son informadas del tema que se va a tratar al comienzo del nuevo proyecto:

"Todos los aprendices escriben de forma independiente una carta dirigida a sus familias informándoles sobre el proyecto que van a realizar y, el tema en el que se van a hacer expertos y sobre el que tienen que investigar en casa". (R.4.1.)

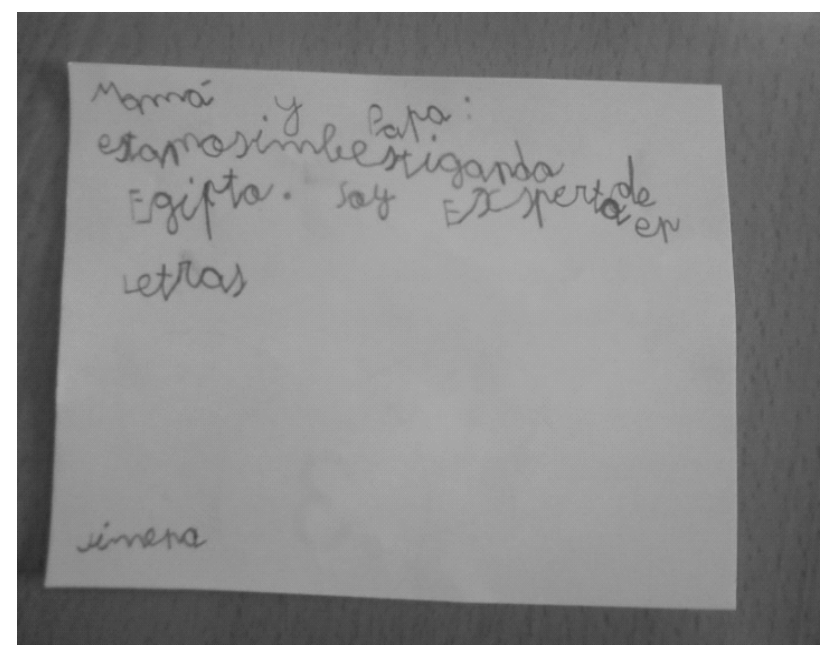

Figura 1. Carta dirigida a las familias informando sobre el nuevo PT. (V.4.1.)

Durante el desarrollo del PT se aprecia una constante relación de comunicación y colaboración familia-escuela, a través de la aportación de información y el apoyo a su hijo o hija en su labor investigadora en casa. Ha de acompañarlo en la búsqueda, comprensión y exposición de la información, que servirá como fuente de aprendizaje para compartir con el resto del grupo:

"- E: ¿Os ayudan papá y mamá a traer información de casa? - Nos ayudan a hacer los trabajos". (E.A.4.2.C.)

"Yo creo que este tipo de enseñanza debería de poner a las madres y decir "pues por aqui tienes que tirar en el caso de que...", por lo menos yo pienso de esa manera". (E.F.4.2.B.) 
"Hasta que ellos ven que es un proceso, que también asumen que tienen que trabajar con ellos en casa para crear una responsabilidad, y que luego ellos vengan y te cuenten, etc. Todo ello requiere un proceso con ellas". (E.M.4.2.)

El proceso de responsabilidad que asumen las familias, en general, es valorado positivamente. El esfuerzo que ponen en juego es recompensado al sentirse copartícipes en el proceso de enseñanza-aprendizaje de sus hijos e hijas. Se sienten integradas en el aula, al concebirse como agentes que tienen la posibilidad de enriquecer con sus aportaciones y conocimientos el tema sobre el que se desea investigar. Sin embargo, existe una gran diferencia entre las actitudes de colaboración de las familias, en función de su disponibilidad horaria y de su voluntad para participar. En los siguientes pasajes expresan sus opiniones:

\section{"Nosotros también formamos parte de su clase". (E.F.4.3.A.)}

"Yo por mí, buenísima, porque, también, es el tiempo que cada madre tenga. Yo me ha pillado en una época que no trabajo y le dedico mucho tiempo a los proyectos, al no trabajar te permite dedicar que cuando Ana dice "una madre voluntaria para ayudarnos"." (E.F.4.3.C.)

"Yo, en mi experiencia, siempre he tenido colaboración, en mayor o menor grado. No se me ha dado la situación de decir "Esto no tira". (E.M.4.3.)

"Resultará enriquecedor pedir la colaboración de las familias para que nos aporten materiales relacionados con el tema".(P.A.4.3.)

Se evidencia que el trabajo por PT mejora la comunicación de las familias con sus hijos e hijas, puesto que constante fluir de información ayuda a intensificar las relaciones. Igualmente, se aprecia que mejoran las relaciones familia-docente puesto que se incrementa la confianza de las familias en la labor educativa que desarrolla la maestra. Las madres y padres tienen la posibilidad de ver y compartir qué se hace en el aula, superando situaciones de enseñanza poco transparentes y casi inaccesibles a las familias:

"Por parte de las familias, la respuesta cuando ellos se sienten incluidos en el proceso de aprendizaje de su hijas y de sus hijos, cuando de verdad los metes en el aula y están allí contigo y con ellos haciendo cosas, la verdad que es una buena forma de relacionarse con ellas". (E.M.4.4.)

"Con este método se comparte mucho lo que es abrir un poquito también, la confianza entre padres e hijo". (E.F.4.4.A.)

"[...] y el niño sabe que tú eres parte de su educación". (E.F.4.4.B.)

Para cerrar esta dimensión, se puede observar que el aula del alumnado siempre está abierta a las familias para participar en cualquier actividad que se proponga. Cuando tienen lugar estas situaciones el alumnado muestra respeto por la persona que entra en el aula y se interesa por los datos que aporta al PT. Se puede observar que los 
aprendices guardan un buen recuerdo de esos momentos, y su aprendizaje persiste en cursos posteriores:

"Viene una sabia de Egipto (un familiar) a la clase y todos los niños y niñas se sientan en la alfombra para escuchar lo que tiene que contarnos". (R.4.5.)

"[...] también, estaban las madres preparándolo en el cuarto donde está A. (las cosas que necesita A.) y, entonces lo trajeron para acá a la clase y hacía tata-ta [...]". (E.A.4.5.B.)

"Siempre hemos tenido confianza para estar en el aula". (E.F.4.5.B.)

En la quinta dimensión se menciona la existencia de una responsabilidad conjunta entre la docente y las familias hacia el aprendizaje del alumnado. Así, en la metodología de PT las familias ejercen una labor de tutorización de sus hijos o hijas haciendo diversas aportaciones en las fases de: documentación, producción y evaluación.

La primera fase en la que las familias realizan aportaciones es la fase de documentación acerca del tema del PT que se esté trabajando. Así, en los resultados se puede apreciar la dedicación de las familias con el alumnado en la búsqueda y obtención de información acerca del tema del proyecto, en este caso, Egipto:

"Ju. habla de su dibujo. Dice que se ha fijado de hojas que le ha sacado su madre del ordenador porque habia cosas que no sabia dibujar". (R.5.1.1.)

"E. también ha traido una hoja con los faraones más importantes de Egipto. Ella va diciendo el nombre de cada uno y su símbolo". (R.5.1.1.)

También, en la fase de producción, las familias median y ajustan la información recogida a las capacidades infantiles para elaborar diversos trabajos que después, el niño o la niña, mostrará y explicará a sus compañeros y compañeras en clase:

"A. enseña su libro de trampas que ha hecho junto a su mamá. Él ha escrito el texto y ha coloreado los dibujos que aparecen. Lo explica con ayuda de la maestra. Sus compañeros y compañeras van añadiendo cosas y comentan lo que va diciendo". (R.5.1.2.)

"Ja. explica el trabajo que ha realizado sobre Egipto. Ha sido una fofucha de Cleopatra. Javier cuenta que es Cleopatra y que está hecha de goma eva. Dice que la han hecho entre su mamá, su hermana y él. Dice que le ha costado hacerlo 4 días". (R.5.1.2.)

Por último, obtenemos aportaciones de la familia en la fase de evaluación del PT. En ella, las familias son partícipes del resultado final del PT, es decir, de los contenidos aprendidos por el alumnado y toman conciencia de su funcionalidad. Esto se muestra a través de la celebración de eventos finales de los proyectos, en este caso, una fiesta de Egipto donde se realizan talleres para la preparación de la misma: 
"Sí que se hace algún evento importante, porque siempre un proyecto tiene una finalidad, aparte de que ellos aprendan lo que quieren aprender, luego al final siempre se prepara algo que tiene un sentido para ellos y que pone en práctica todo lo que han aprendido. Entonces, un poco una funcionalidad". (E.M.5.1.3.).

"Siempre que hemos terminado un proyecto, siempre hemos hecho algo especial". (E.F.5.1.3.C.)

"Con las familias: Talleres; Fiesta final: fiesta de faraones [...]". (P.P.5.1.3.)

"Elaboración del disfraz de faraones. Por un lado, unas mamás se encargan de hacer el disfraz con bolsas de basura blancas y por otro, otras se distribuyen entre las manchitas para ayudar a los niños y niñas a elaborar los collares para el disfraz". (R.5.1.3.)

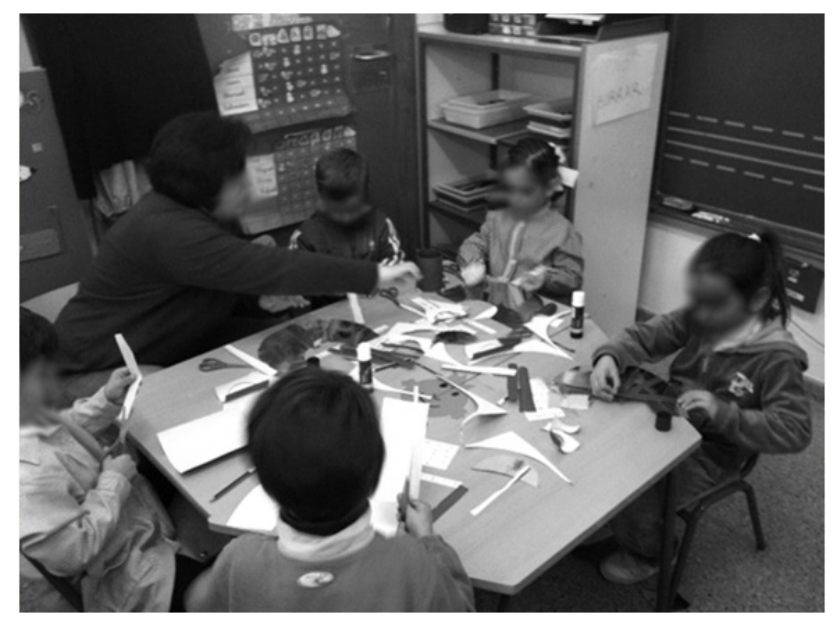

Figura 2. En la fotografía se muestra a una mamá ayudando a los discentes a realizar collares para el disfraz de faraones y faraonas. (V.5.1.3.)

En la sexta y última dimensión, se pueden apreciar los beneficios que produce el trabajo por proyectos desde el punto de vista familiar. Se apreciala curiosidad y estimulación generadas por el PT en el aprendiz. Además, las familias exponen que el método de PT permite que el alumnado cometa errores y se aprovecha la denominada 'pedagogía del error', aceptando los errores como elementos fértiles de aprendizaje, haciéndoles comprender el sentido y la coherencia de lo que aprenden.

"[...] Yo lo que le veo importante es el concepto general de lo que es el proyecto. No es solamente lo que nosotros aportemos. Yo creo que a Ana le daría exactamente igual que le trajéramos cuatro papeles siempre y cuando los niños supieran qué tienen que hacer con ellos". (E.F.6.1.A.) 
"Ellos aprenden que se pueden equivocar. Si tú te equivocas, eso forma parte de tu aprendizaje. De la otra manera (método tradicional), el error es castigo". (E.F.6.1.A.)

"Eso también es una cosa que han aprendido aquí. A decir "por qué", "pero ¿por qué esto es así?" y tú le dices porque si y el te dice "porque sí, no. Porque las cosas son por algo. Porque mi seño Ana dice que las cosas son por algo". (E.F.6.1.B)

En los resultados, también observamos ciertas limitaciones de los PT expuestas por las familias. Consideran que es un método que requiere mucha dedicación de tiempo de la familia con el aprendiz, algo que, actualmente, es difícil debido al ajetreado ritmo de vida imperante. También, las familias afirman que con los PT, a veces, se encuentran desorientadas a la hora de dar pautas para la tutorización del aprendizaje de sus hijos e hijas en casa. Por último, encontramos que los aprendizajes obtenidos con los PT a largo plazo, generan cierta impaciencia en las familias.

"El problema del método, el que yo veo, es que todo el mundo no tiene el mismo tiempo para dedicarle al hijo" (E.F.6.2.A.)

"[...] como tú no sabes por dónde tirar, porque como no estás acostumbrada y no saber por dónde tirar pues la primera vez que te lo dice, pues dices "¿cómo le voy yo a hablar a este niño con 3 años de cómo va la sangre?"'. (E.M.6.2.B.)

"[...] fijate que estamos en el $2^{\circ}$ trimestre, y es cuando yo le estoy viendo muchísimos resultados, pero ahora. Cuando están en 3 años, en 4 años o empiezan, incluso, 5 años, tú lo ves todavía que dices "madre mía...", que no entiendes, a lo mejor por dónde los niños van". (E.F.6.2.C)

"[...]mi otra hija, [...]sabía leer con 4 años, sabía escribir en minúscula al final de 4 años también, y creía que con este método no se iba poder conseguir porque no veía fichas, no veía resultados. Sin embargo, no veía lo que estaba trabajándose. No lo conocía, entonces yo me vine a habar con Ana, me explico y me dijo "dale tiempo y dale tiempo" y mi niña con 4 años sabía leer". (E.F.6.2.A.)

Para concluir esta dimensión, se menciona una sugerencia en la que insisten las familias de forma reiterativa. Se refieren a la necesidad de continuar con el trabajo por PT en las etapas posteriores a Educación Infantil. Algunas familias recriminan la enorme descoordinación que existe entre la educación que se recibe en la etapa de Infantil y de Primaria, no teniendo esta última ninguna continuidad con respecto a la primera. Las familias acusan y se quejan del gran cambio al que se ven sometidos sus hijos e hijas, debido a que la nueva etapa no conserva ninguna característica de la anterior.

"Creo que debería estar lo que es la Educación Primaria y la Educación Infantil mucho más ligada y preguntarle mucho más a los profesores de Infantil 
como van esos niños; mucha más coordinación interna, que falta coordinación porque luego ya te exigen que todos lleguen igual, que todos tengan los mismo conocimientos, te imponen los temarios, te imponen lo que tienes que saber acerca de algo, tú no lo descubres". (E.F.6.3.A.)

\section{Discusión y conclusiones}

Esta investigación muestra que la metodología de PT facilita la inclusión y participación de las familias en el desarrollo de los PT. Utilizamos como eje vertebrador de las conclusiones los objetivos establecidos en este estudio.

En primer lugar los conocimientos y experiencias profesionales de las familias son aprovechadas en la metodología de PT porque suponen el 'alimento' que ofrecen a sus hijos e hijas en sus hogares, constituyendo de este modo el bagaje experiencial y la actitud ante el conocimiento que los discentes muestran en clase (Muntaner, 2000). De esta manera, los datos confirman que existen diferentes niveles de motivación, variedad de intereses y diversos ritmos de aprendizaje en el aula, que se ponen de manifiesto en el método de PT. Esta metodología potencia la expresión libre del alumnado para narrar sus experiencias familiares y vitales, poniendo en marcha situaciones educativas donde la heterogeneidad de vivencias, experiencias y emociones constituye la esencia de la vida del aula. No se producen procesos educativos uniformantes que acallen, bajo la cumplimentación de materiales comerciales comunes y descontextualizados, la diversidad del alumnado y de las familias que colaboran en su formación.

Tal como revelan los datos, las familias entrevistadas tienen la oportunidad de ofrecer sus saberes al aula, la cual recibe y valora sus aportaciones al asumir una concepción corresponsable de la labor educativa. De este modo se incorporan desde las experiencias de las familias (elemento desencadenante del PT de Egipto), así como sus saberes y experiencias profesionales al proceso educativo, constituyendo la esencia de las situaciones de enseñanza-aprendizaje. Coherente con otros estudios (Torío, 2004 y Yuen, 2009), nuestro trabajo muestra que el alumnado, en este caso, posee más conocimientos previos del tema de lo que la docente espera

La comunicación fluida entre docente y familia es muy importante para que su relación sea positiva (Valdés, Martín y Sánchez-Escobedo, 2009). Así, los resultados obtenidos revelan que los PT consideran a las familias parte del proceso de enseñanzaaprendizaje desde el principio. Les hacen partícipes de cada una de las fases, de todo lo que ocurre durante el PT, perfilándose nítidamente los roles de corresponsabilidad que han de asumir.

El segundo objetivo hace referencia a la tutorización o acompañamiento de las familias en el proceso educativo de sus hijos e hijas. En efecto, en el PT observamos que las familias, además de colaborar en la búsqueda, selección y suministro de información, sobre todo han de responsabilizarse de tutelar el aprendizaje de los niños y niñas en casa. Las familias asumen el papel de mediación entre las capacidades infantiles y los nuevos materiales de aprendizaje. De este modo, facilitan aprendizajes 
significativos al establecer un puente entre las ideas previas y el nuevo material de aprendizaje En realidad, las familias asumen el desafío de situarse en la Zona de Desarrollo Próximo (Vygotsky, 2012), lo cual en ocasiones les genera cierta incertidumbre al no saber cómo ofrecer una ayuda pedagógica ajustada a las demandas de sus hijos e hijas. Esta tutela en el aprendizaje que las familias asumen supone un efecto multiplicador en los aprendizajes, puesto que además de hacer protagonistas a sus criaturas, les ofrecen la oportunidad de incrementar la motivación que supone compartir sus hallazgos con los compañeros y compañeras. Del mismo modo, experimentar las dificultades de liderar una situación educativa les enseña a valorar más la labor de la docente de Infantil (López Melero, 2011).

El siguiente objetivo planteado alude al análisis de las actividades en las que las familias colaboran. Los datos muestran nítidamente cómo las familias están presentes desde el inicio hasta que concluye el PT. Comienza su presencia en la carta elaborada para solicitar su participación por parte de los niños y niñas, continúa por sus aportaciones de materiales e información relevante para construir el rincón del PT, sigue con su colaboración en actividades como el experto o los talleres y concluye con su participación en la fiesta final del PT Lo más significativo no es su presencia transversal en todo el PT, lo más relevante es la calidad de su participación. Se aleja de una colaboración puntual o esporádica, para convertirse en una verdadera corresponsabilidad en el proceso de enseñanza-aprendizaje, sin cuya existencia sería inviable la aplicación de este método. Por tanto, la participación familiar se configura como un elemento sustantivo e imprescindible de los PT que demanda una visión abierta de la educación y una concepción de inteligencia distribuida, en donde no solo prevalece el conocimiento experto del docente, sino que por el contrario todas las personas que conforman la comunidad educativa tienen cosas que enseñar y que aprender.

Para finalizar nos interesamos por clarificar las ventajas y limitaciones que, según las familias, se derivan del método de PT. En este sentido los datos reflejan que las familias son conscientes de los beneficios obtenidos con los PT en el aprendizaje de sus hijos e hijas, pero también exponen ciertas limitaciones acerca de esta metodología, proponiendo algunas sugerencias como elementos de mejora. Mérida et al., (2011) nos muestran que en los PT se aceptan todas las ideas iniciales del alumnado, y que a medida que avanza el PT y van experimentando nuevas situaciones de enseñanzaaprendizaje el alumnado toma conciencia de los errores existentes en sus hipótesis de partida. De este modo, los resultados obtenidos muestran principios inclusivos en los PT a través de la aceptación de los errores de los aprendices y su posterior validación o rechazo mediante la experimentación. Los resultados muestran que las familias consideran muy beneficioso la consideración de las opiniones de los niños y niñas, aunque sus ideas no sean completamente ajustadas, procurando que sean conscientes del proceso racional que implica la adquisición de cualquier nuevo aprendizaje.

La limitación de los PT más subrayada por las familias es la descoordinación existente entre la metodología empleada en Educación Infantil y Primaria, especialmente intensa, cuando en Educación Infantil se trabaja por proyectos, dado que en su mayoría están más familiarizadas con el método tradicional. También señalan 
que el tiempo de dedicación que requieren los PT, así como la preparación que deben poseer las familias para poder colaborar en el proceso de documentación y producción de los PT que se realiza en casa es, a veces, demasiado elevado. Esto puede generar diferentes niveles de compromiso e implicación en el aula, lo cual puede resultar perjudicial para aquellos niños y niñas cuyas familias puedan dedicar menos tiempo a la preparación de materiales, y por tanto, se pueden sentir un poco segregados de la dinámica del aula.

Por último, las familias insisten en que debe haber una continuidad y coherencia entre la etapa de infantil y primaria evitando discontinuidades y saltos bruscos que afecten negativamente a la evolución normal de los discentes. Las familias constatan por experiencias propias y ajenas que el alumnado al acabar la etapa infantil sufre un cambio de gran magnitud, experimentando estilos de aprendizaje radicalmente diferentes. Por eso, es importante el trabajo conjunto entre los docentes de ambas etapas, sin vulnerar el desarrollo iniciado en Infantil y haciendo que el alumnado viva esta transición sin grandes dificultades (Galán y García, 2008).

Para concluir no queremos dejar de significar la prudencia con la que hay que interpretar estas conclusiones puesto que somos conscientes de que una de las limitaciones de este trabajo es el reducido número de familias entrevistadas. Dicha limitación, aunque se ha tratado de paliar utilizando la triangulación metodológica con otros instrumentos de recogida de información como la documentación pedagógica, supone un resorte para seguir profundizando en las percepciones familiares respecto a la metodología de PT. Como mirada de futuro nos proponemos indagar las percepciones que poseen las familias del alumnado con necesidades educativas específicas respecto a la capacidad inclusiva del método de PT.

\section{Referencias bibliográficas}

AGUADO IRIBARREN, L. (2010). Escuela inclusiva y diversidad de modelos familiares. Revista Iberoamericana de Educación, 53(6), 1-11.

AGUADO ODINA, T. (2010). Diversidad e igualdad en educación. Madrid: UNED.

AINSCOW, M., BOOTH, T. Y DYSON, A. (2004): "Understanding and developing inclusive practices in schools: a collaborative action research network". International Journal of Inclusive Education, 8(2), 125-139.

ARNÁIZ, P. Y DE HARO, R. (2004). Ciudadanía e interculturalidad: claves para la educación del siglo XXI. Educatio, 22, 19-37.

BANKS, M. (2010). Los datos visuales en investigación cualitativa. Madrid: Morata.

BARRIO, J. L. (2009). Hacia una Educación Inclusiva para todos. Revista Complutense de Educación, 20(1), 13-31.

BENITO, P. Y BALLESTEROS, B. (2012). Diversidad cultural, eficacia escolar y mejora de la escuela: encuentros y desencuentros. Revista de Educación, 358, 1737. 
BOOTH, T. AINSCOW, M. Y KINGSTON, D. (2006). Index para la inclusión. Desarrollo del juego, el aprendizaje y la participación en Educación Infantil. Bristol, Reino Unido: Ed. Centre of Inclusive Studies on Inclusive Education.

DÍEZ, C. (2002). La oreja verde de la escuela. Trabajo por proyectos y vida cotidiana en la escuela infantil. Madrid: De la Torre.

DOMÍNGUEZ, G. (2003). En busca de una escuela posible. Revista Interuniversitaria de Formación del Profesorado, 17(3), 29-47.

ECHEÍTA, G. Y SANDOVAL, M. (2002). Educación inclusiva o educación sin exclusiones. Revista de Educación, 327, 31-48.Flick, U. (2004). Introducción a la investigación cualitativa. Madrid: Morata.

GALÁN M.J., Y GARCÍA ESPINOSA, A. (2008). El tránsito de educación infantil a primer ciclo de primaria.P@k en redes, Revista Digital,3(1), 1-28. Extraído el 13 de julio de 2013 de http://pakenredes.cepalcala.org.

GALLARDO, I.M. (2013). Construcción del conocimiento en Educación Infantil. Revista Iberoamericana de Educación, 63(1), 1-7.

GIBBS, G. (2012). El análisis de datos cualitativos en investigación cualitativa. Madrid: Morata.

GUIJO, V. (2008). Derechos de la infancia (0-6 años) y educación inclusiva. Revista de Educación, 347, 55-74.

HERNÁNDEZ, F. (2000). Los Proyectos de Trabajo: la necesidad de nuevas competencias para nuevas formas de racionalidad. Educar, 26, 39-51.

HERNÁNDEZ, F. (2002). Los Proyectos de Trabajo. Mapas de navegantes en mares de incertidumbre. Cuadernos de Pedagogía, 310, 78-82.

LÓPEZ, F., DÍEZ, M., MORGADO, B. y GONZÁLEZ M.M. (2008). Educación infantil y diversidad familiar. XXI, Revista de Educación, 10, 111-122.

LÓPEZ MELERO, M. (2011). Barreras que impiden la escuela inclusiva. Innovación Educativa, 21, 37-54.

LUENGO, J.J., LUZÓN, A. (2001). El proceso de transformación de la familia tradicional y su implicaciones educativas. Investigación en la Escuela, 44, 55-68.

MÉRIDA, R., BARRANCO, B., CRIADO, E., FERNÁNDEZ, N., LÓPEZ, R. M. Y PÉREZ, I. (2011). Aprender investigando en la escuela y en la universidad. Una experiencia de investigación-acción a partir del Trabajo por Proyectos. Investigación en la escuela, 73, 65-76.

MÉRIDA, R., GONZÁLEZ, E. Y OLIVARES, M.A. (2015). La creación de entornos educativos inclusivos en Educación Infantil a través de proyectos de trabajo. I Congrés Cientific Internacional: Aprenentatges i interaccions a l'aula. Valencia, 13 y 14 de marzo. 
MUNTANER, J.J. (2000). La igualdad de oportunidades en la escuela de la diversidad. Profesorado, Revista de Currículum y Formación de Profesorado, 4(1), 1-17.

MUNTANER, J. J. (2010). De la integración a la inclusión: un nuevo modelo educativo. En Arnáiz, P.; Hurtado, Ma.D. y Soto, F.J. (Coords.) 25 Años de Integración Escolar en España: Tecnología e Inclusión en el ámbito educativo, laboral y comunitario. Murcia: Consejería de Educación, Formación y Empleo.

PARRILLA, M.A. (2002). Acerca del origen y sentido de la educación inclusiva. Revista de Educación, 327, 11-29.

RUIZ DE MIGUEL, C. (1999). La familia y su implicación en el desarrollo infantil. Revista Complutense de Educación, 10(1), 289-304.

SALES, A. (2004). Hacia una escuela inclusiva intercultural: los proyectos de trabajo como propuesta curricular y formativa. XXI, Revista de Educación, 6, 139-153.

SÁNCHEZ NÚÑEZ, C.A. Y GARCÍA GUZMÁN, A. (2009). Implicación de las familias en una escuela intercultural: una visión compartida. REI, Revista de Educación Inclusiva, 2 (2), 11-29.

TORÍO, S. (2004). Familia, Escuela y Sociedad. Aula Abierta, 83, 35-52.

VALDÉS, A.A., MARTÍN, M. Y SÁNCHEZ-ESCOBEDO, P.A. (2009). Participación de los padres de alumnos de educación primaria en las actividades académicas de sus hijos. Revista Electrónica de Investigación Educativa, 11, 1, 1-17. Extraído el 2 de febrero de 2014, de http://redie.uabc.mx/index.php/redie.

YUEN, L.H.F. (2009). From Foot to Shoes: Kindergartners', Families' and Teachers' Perceptions of the Project Approach. Early Childhood Education Journal, 37, 2333.

VYGOTSKY, L.S. (2012). El desarrollo de los procesos psicológicos superiores. Barcelona: Crítica.

ZABALA, A. (2012). La práctica educativa. Cómo enseñar. Barcelona: Graó.

\section{Correspondencia con los autores}

María del Carmen RUIZ TORRES

e-mail: mcarmen.ruiz4@gmail.com

Rosario MÉRIDA SERRANO

Rectorado de la Universidad de Córdoba.

Avda Medina Azahara, no 5. CP 14071. Córdoba.

e-mail: ed1meser@uco.es 